
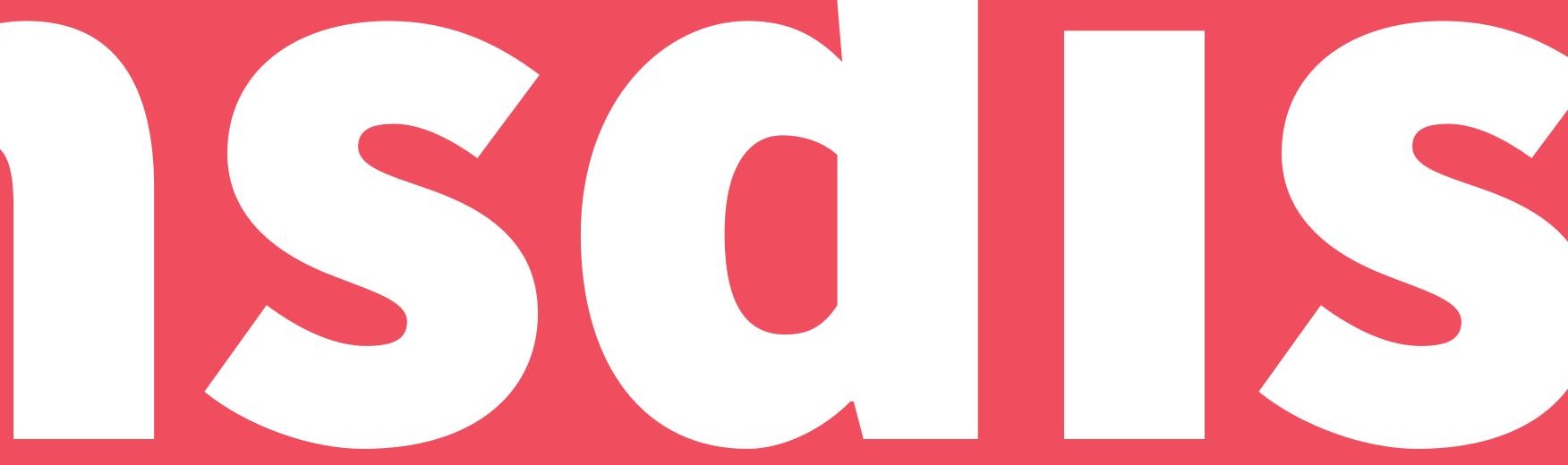

\title{
Innovación transdisciplinar en la enseî́anza del Diseño de Vestuario
}

\section{Resumen}

El presente artículo trata sobre el uso e impacto de diferentes tecnologías en el diseño de vestuario y los aportes brindados por otras disciplinas a través de un diálogo inter y transdisciplinar. Para realizar esta reflexión se emplea como herramienta metodológica el estudio de caso de la asignatura Taller 6, del Programa Diseño de Vestuario, de la Universidad de San Buenaventura, Cali, para observar la implementación de nuevas tecnologías en la enseñanza del diseño de vestuario y los aportes realizados a las

\section{Edgar Franco} Medina $^{1}$

Rosmery Dussán Aguirre 2

\section{Nancy Otero}

\section{Buitrago 3}

${ }^{1}$ Universidad de San

Buenaventura, Cali,

Colombia,efranco@

usbcali.edu.co

${ }^{2}$ Universidad de San

Buenaventura, Cali,

Colombia, director.

disenovestuario@usbcali.

edu.co

${ }^{3}$ Secretaria de Educación

Municipal, Cali, Colombia, oterobn16@yahoo.es

https://doi.org/10.53972/

RAD.erad.2021.2.10 
Edgar Franco Medina

Rosmery Dussán Aguirre

Nancy Otero Buitrago

estudiantes en su formación profesional, de acuerdo con las competencias requeridas en este campo del saber, de cara al siglo XXI.

Palabras clave

Transdisciplinar, diseño de vestuario, tecnologías, pedagogía. 
Introducción

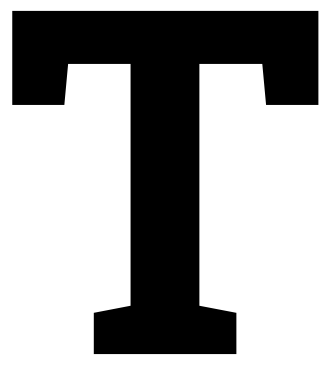

Teniendo en cuenta la importancia de pensar y analizar las prácticas docentes en el aula y la función de enseñar, a partir de las preguntas: ¿qué se enseña?, ¿cómo se está enseñando? y ¿para qué se enseña?, tomando como referente el trabajo realizado en programas de educación superior, como el Programa de Diseño de Vestuario, ofrecido por la Universidad de San Buenaventura, Sede Cali, Colombia, el propósito de este artículo es realizar una reflexión pedagógica, sobre el uso e impacto que tiene actualmente las tecnologías en la enseñanza del diseño de vestuario y los aportes que brindan otras disciplinas, en el marco de un diálogo intery transdisciplinar, como estrategias claves de formación para las estudiantes, de acuerdo con su perfil profesional, las necesidades del entorno y de la sociedad globalizada. El Programa de Diseño de Vestuario, es un programa innovador y vanguardista, que enseña a las estudiantes a desarrollar conceptos, a partir del estudio y análisis de su entorno, para aprender a elaborar prototipos de diseño, por medio de procesos de consulta, trabajos de laboratorio e investigación aplicada, que pueden llevarlas a crear posibles colecciones, utilizando el diseño, la ciencia y la tecnología como herramientas claves de su formación como profesionales. 


\section{Marco teórico}

Para el desarrollo de esta propuesta de innovación, aplicada al diseño de vestuario, se ha tenido en cuenta el falsacionismo popperiano, que indica que el objetivo de la ciencia consiste en encontrar la explicación de hipótesis, mientras que la tecnología busca artefactos confiables (Oliveras, 2010). En este sentido, en el diseño de vestuario se toma como referente el proceso de pensamiento de diseño a través del cual se logra el desarrollo de la creación de indumentaria que satisfaga las necesidades de un usuario (Darbellay, Moody y Lubart, 2017). Para ello se requiere un acercamiento con una muestra de la población objetivo para determinar sus necesidades y requerimientos; lo que llamamos realización de un trabajo de campo (Ovalle, 2014).

La innovación en la enseñanza del diseño de vestuario, tiene como referente un enfoque transdisciplinar, que consiste en utilizar elementos trasversales al diseño, haciendo uso de las nuevas tecnologías, así como otras disciplinas entre ellas la ética y las estéticas contemporáneas, para que las estudiantes alcancen una formación integral, con responsabilidad social y medioambiental, de acuerdo con el tipo de profesionales que demanda la sociedad actual y de cara al futuro, en el marco de la sostenibilidad.

Otra categoría de análisis aplicada al diseño de vestuario es el pensamiento complejo, distante de la visión reduccionista y de separación de los saberes, lo que permite que dicha resolución se planteé como un sistema complejo (Tarride, 1995). Este abordaje, permite interrelacionar e intercomunicar las diferentes disciplinas, encontrando una sinergia entre especialidades, metodologías y en algunos casos tecnicismos de cada disciplina, que mejoren la comunicación efectiva entre las mismas (Barberousse Alfonso, 2008). 
Descripción de la innovación

En estos proyectos no solo se consideran los aspectos y variables estrictamente de las disciplinas interrelacionadas en la solución del problema, sino que permite a las estudiantes realizar una mirada holística, donde la metodología del diseño, aparte de definir requerimientos ergonómicos, funcionales, entre otros, también deberá considerar requerimientos legales, políticos, sociales, ambientales, estéticos, económicos, que permitan abordar la resolución del problemas desde una mirada transdisciplinar que aplica teorías, conceptos y métodos de otras disciplinas con el objetivo de desarrollar una síntesis global (Lattuca, 2003).

De acuerdo con la metodología del Programa, se pretende desarrollar un enfoque interdisciplinar y transdisciplinar, en cursos como los "Talleres" que en total son seis, en el transcurso de la carrera universitaria. Estos Talleres, son orientados por docentes formados en el campo del Diseño de Vestuario, el Diseño Industrial, y la Ingeniería de Materiales, entre otros, que tienen como finalidad generar las condiciones de posibilidad, que permita a los y las estudiantes, adquirir herramientas teóricas y prácticas, para que puedan utilizarlas funcionalmente y de manera autónoma, en su formación como diseñadoras y posteriormente como profesionales.

En la metodología del Programa, se tienen en cuenta los aportes que ofrece el diálogo establecido entre diferentes campos del saber, como la ergonomía, innovación, sostenibilidad, comunicación, la estética y el saber pedagógico, entre otros campos, que van orientados a brindar las herramientas teórico-prácticas de la enseñanza del diseño de vestuario, para que las estudiantes logren apropiarse de conocimientos de diferentes disciplinas y con ello, realicen trabajos creativos e innovadores en su campo de desempeño. El enfoque interdisciplinar que se aplica en los Talleres pretende, además, hacer converger varias disciplinas, haciendo uso de diferentes 
tecnologías, para la resolución de problemas de diseño, que no se pueden resolver sólo desde la teoría del vestuario como objeto de investigación. Por tal motivo, se tiene en cuenta la interacción que existe entre el sujeto, el vestuario y su entorno, desde una mirada global, holística y contextualizada, que permite enfocar una solución coherente y funcional, frente los problemas de estudio e investigación a resolver.

\section{Proceso de implementación de la innovación}

El diálogo interdisciplinar que se establece entre el Diseño de Vestuario y otras disciplinas surge a partir del análisis de las tendencias relacionadas con el uso de tejidos tecnológicos inteligentes, que brindan funciones adicionales al usuario a través del vestuario. Por ejemplo, con el uso de la tecnología se espera que los vestuarios desarrollados por las estudiantes sean funcionales, brindando beneficios estéticos, cosméticos, estructurales e incluso emocionales. La finalidad de la metodología del curso es que los y las estudiantes puedan apropiarse de los conocimientos requeridos para que hagan uso de las diferentes tecnologías, con el fin de resolver problemas que involucren aspectos sociales, políticos, éticos, estéticos, culturales, entre otros. En algunos proyectos desarrollados, las estudiantes no encuentran específicamente los elementos que ayuden a resolver su problema de diseño de vestuario y pasan a realizar investigación científica interdisciplinar, la cual desarrollan con estudiantes de otras disciplinas como la química, microbiología, ingeniería electrónica, ingeniería de materiales, entre otras. Por ejemplo, si en determinado proyecto, un requerimiento consiste en microencapsular un aroma especifico, pero este no se encuentra disponible en el mercado, deberán plantear la hipótesis de la posibilidad, o no, de crear el aroma a partir de esencias y luego microencapsularlo a través de prácticas de laboratorio con la asesoría de profesionales expertos en química 
de materiales, y así encontrar la solución a los requerimientos de manera más precisa. Vale la pena mencionar, que en este ejemplo especifico, el beneficio que obtendrá el usuario, con la intervención del textil con aromas microencapsulados, será el de alcanzar una mayor duración del olor, aun después de varios ciclos de lavado. En este proceso de desarrollo del proyecto de vestuario, luego de tener claros la totalidad de los elementos de diseño, las estudiantes desarrollan un prototipo, con la tecnología aplicada, que, si es viable, puede llevar a la creación de una colección de vestuario. Como caso puntual se puede observar en la asignatura Taller 6, un énfasis en tecnologías aplicadas al diseño de vestuario. En este taller, se enseña teniendo como base la pedagogía activa, en donde las estudiantes son partícipes centrales en su proceso de aprendizaje, así que se les ofrecen herramientas teóricas, para que, a través de estas, y por medio de actividades prácticas, las estudiantes logren hacer uso de diferentes tecnologías, básicas y avanzadas, provenientes de otras disciplinas, para aplicarlas al diseño de vestuario. En este curso, se hace un análisis de los diferentes materiales que utiliza la Ingeniería de Materiales, la cual aporta en la selección, caracterización y desarrollo de textiles que son aplicados en sus prototipos de vestuario y colecciones, debido a que en algunos casos las estudiantes diseñan un conjunto de prendas relacionadas con un concepto específico de diseño.

Los procesos de diseño de vestuario que se desarrollan en este taller se apoyan en los aportes teóricos y prácticos de otras disciplinas como la Ingeniería Agroindustrial, que permite el uso de materiales reciclados de origen natural y renovables, lo cual le brinda herramientas para que los procesos desarrollados a partir de este campo, sean sostenibles. La Ingeniería Electrónica, por su parte, permite la incorporación y adaptación de dispositivos electrónicos como microprocesadores, luces led, sensores, entre otros, que convierten el vestuario en interactivo y funcional. 
En el Taller 6, se desarrollan, además, prácticas en laboratorios de química, microscopía, materiales, electrónica, diseño, patronaje, confección y Fab Lab, o laboratorio de elaboración de prototipos. Así mismo, se ha implementado la práctica de electrohilado de nanofibras poliméricas, que se enmarca en el campo del desarrollo de nanomateriales a través de la nanotecnología(Franco, Dussán, Amú, \& Navia, 2018). Este campo ha permitido desarrollar nanofibras de PVA y de nilón respectivamente, con el fin de que las estudiantes tengan un acercamiento al uso de estructuras nanométricas, a través del conocimiento de los procesos y técnicas de microscopía.

Las estudiantes han aprendido a usar también, la técnica de microencapsulación, a través de levadura, lo cual ha permitido microencapsular diferentes principios activos como aromas, donde el vestuario cumple funciones de aromaterapia para generar una sensación de relajación y desestresante al usuario. Otras sustancias como el Aloe vera, son microencapsuladas y adheridas al textil, con la finalidad de hidratar la piel del usuario. Además, se han microencapsulado principios activos antibacteriales, que cumplen una función de inhibir la proliferación de bacterias en algunas partes del cuerpo, para evitar malos olores. A través de la enseñanza de los E-textiles, los textiles se hacen funcionales con dispositivos electrónicos, con el fin de que a partir de estímulos externos se activen sensores para encender y apagar luces led, servomotores, sonidos, entre otros dispositivos. El uso de esta tecnología conlleva a la interactividad del usuario con los diferentes dispositivos, articulados con tecnologías de la informática, pues en algunos casos se conectan con dispositivos móviles, como los celulares, por medio de bluetooth o wifi. Las prácticas específicas que se desarrollan en este laboratorio, consisten en la conexión y programación de una luz led, con un microprocesador Arduino Lilypad. Adicionalmente, se trabaja con sensores de luz, temperatura, buzzer, y viber. A las estudiantes se les enseña a integrar estos dispositivos a sus diseños, a través de 
hilos metálicos y agujas a las telas, teniendo en cuenta los aspectos ergonómicos que se deberán considerar en sus diseños de vestuario.

En la práctica de la Microscopia electrónica de barrido (SEM), las estudiantes y docentes, seleccionan siete muestras de materiales, principalmente textiles, aunque en algunos casos, se han llevado otras muestras biológicas para su observación. Posteriormente, las muestras son recubiertas con una capa de oro y llevadas al microscopio. Las imágenes obtenidas, que para esta técnica se obtienen en escala de grises, son editadas y coloreadas por las estudiantes con los programas Illustrator y Photoshop, con el fin de estamparlas sobre las telas, a través de la técnica de sublimación. Esto ha llevado a que los diseños gráficos de dichos estampados sean únicos, dándole originalidad a la prenda.

\section{Evaluación de resultados}

Para que el proceso de diseño sea efectivo, siguiendo esta metodología, los diseñadores de vestuario, e ingenieros deben conocer los términos y tecnicismo básicos de la disciplina o disciplinas distintas a la propia, que están presentes en la resolución del problema de Diseño. Se podrá considerar este caso como un sistema complejo que conlleve a una relación transdiciplinar, si se tiene en cuenta la mayor cantidad de disciplinas dentro del problema, por ejemplo, ergonomía, innovación, estética, etc., teniendo en cuenta su funcionalidad. También se tiene encuenta aspectos sociales, ambientales, económicos, éticos e incluso de carácter moral, que permitirán esa mirada holística, característica de los sistemas complejos.

A continuación, se presentarán dos casos en los cuales se ha aplicado la metodología en mención:

La Figura 1, presenta una imagen obtenida con un microscopio electrónico de barrido (SEM), de las Microcapsulas de levadura con 
En el otro caso, relacionado con la temática de los E-Textiles, las estudiantes Gloria Lizeth Camayo y Natalia Riaño en su proyecto de curso, desarrollaron en conjunto con estudiantes de ingeniería electrónica, un vestuario para niños invidentes con sensores de distancia por ultrasonido (módulos HC-SR04), controlados por Arduino. Estos dispositivos detectan obstáculos e indican a los niños, a través de ruidos y vibración, la cercanía de los mismos. La disposición de los sensores en el vestuario fue en la parte frontal de la chaqueta y el gorro, este último para prevenir que el niño reciba golpes en su rostro o cabeza, como se puede observar en la Figura 2.

Figura 2 Vestuario para niños Invidentes con detector de obstáculos

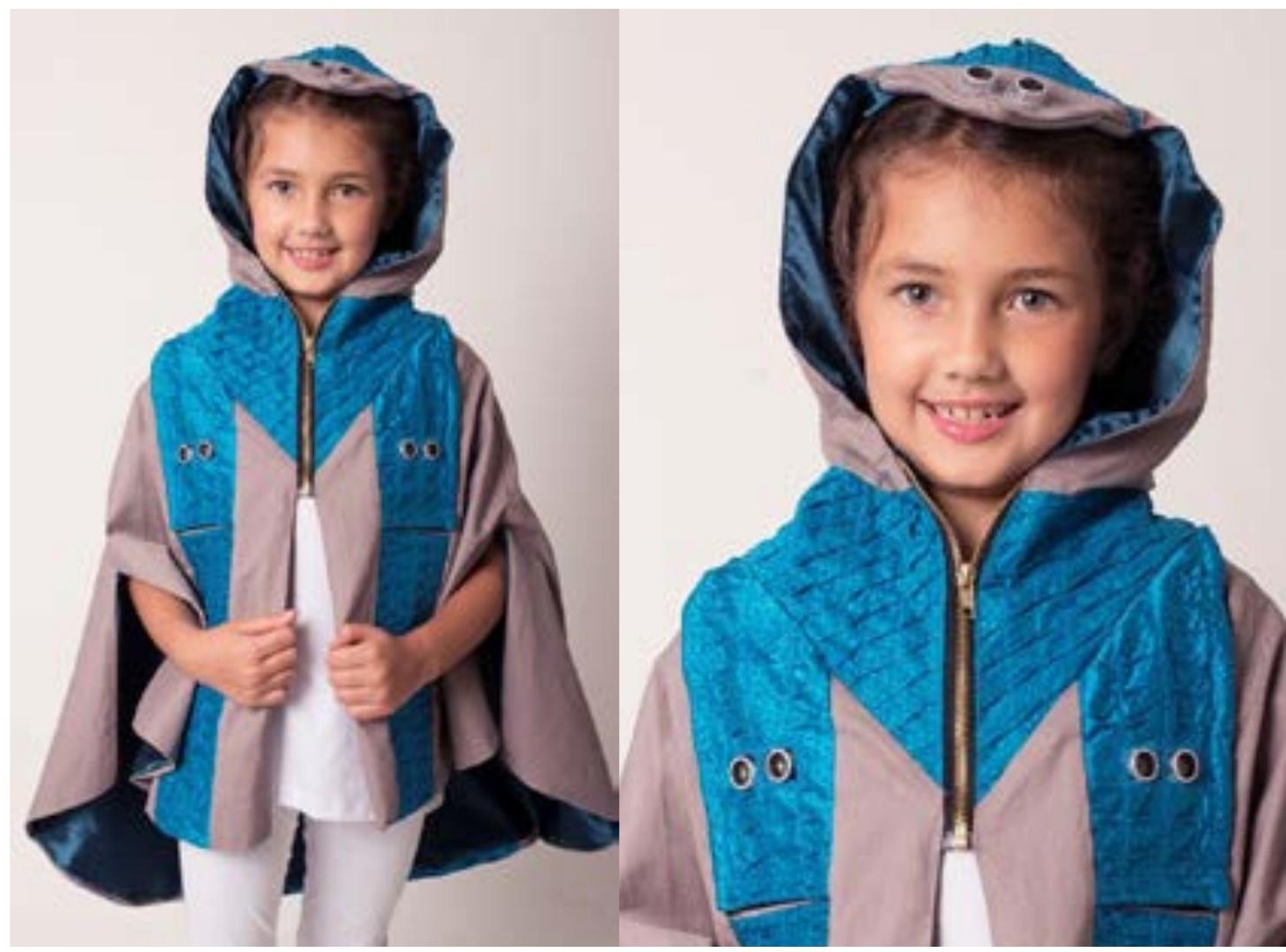




\section{Conclusiones}

Los anteriores son algunos de los ejemplos representativos, del trabajo que se puede realizar en el aula de clase y fuera de ella, en los laboratorios, tomando como muestra algunas de las prácticas que se desarrollan en el Taller 6, del Programa de Diseño de Vestuario. Las clases que se imparten tienen como finalidad que las estudiantes alcancen los logros esperados por el Programa de Curso, que les brinde herramientas significativas y funcionales, en su formación como diseñadoras de vestuario. Esto se logra alcanzar a través del diálogo interdisciplinar y transdiciplinar que se realiza para fortalecer el proceso de enseñanza - aprendizaje, de las estudiantes, utilizando herramientas de otros campos del saber, para que ellas logren incorporarse profesionalmente en el mundo laboral y social que depara su presente inmediato, y de acuerdo al tipo de sujeto con las competencias requeridas en su campo de formación, apoyadas por los avances de la ciencia y la tecnología, de acuerdo con los requerimientos sociales, políticos y culturales que demanda el siglo XXI.

\section{Referencias bibliográficas}

Barberousse Alfonso, P. (2008). Fundamentos teóricos del Pensamiento complejo de Edgar Morin. Revista Educare, XII(1994), 95-113. DOI: https://doi.org/ISSN: 1409-42-58

Darbellay, F., Moody, Z., \& Lubart, T. I. (2017). Creativity, Design Thinking and Interdisciplinarity. Singapur: Springer.

Franco, E., Dussán, R., Amú, M., \& Navia, D. (2018). Statistical Optimization of the Sol-Gel Electrospinning Process Conditions for Preparation of Polyamide 6/66 Nanofiber Bundles. Nanoscale Research Letters, 13(1), 230. DOI: https://doi.org/10.1186/ s11671-018-2644-9 
Lattuca, L. R. (2003). Creating Interdisciplinarity: Grounded Definitions from College and University Faculty, History of Intelectual Culture, 3(1). Recuperado de: https://www.ucalgary.ca/hic/files/ hic/lattucapdf.pdf

Oliveras, J. M. (2010). Ciencia, tecnología y diseño. Diseño Y Sociedad. Recuperado de: http://skat.ihmc.us/rid=1460491285146_145946 8040_321/RELACION CIENCIA TECNOLOGIA Y DISEÑO.pdf

Ovalle, M. A. (2014). Compasáureo: investigación y proyecto de diseño. Revista KEPES, 10, 317-334.

Tarride, M. (1995). Complejidad y Sistemas Complejos. MANGUINHOS, II. Retrieved from http://uazuay.edu.ec/analisis/Complejidad y Sistemas Complejos.pdf 
